aromas sobrevivieron en un radio de 300 metros en la dirección del viento. Fuentes y sus colegas reportaron estos hallazgos en la revista Atmospheric Environment, vol. 42, No 10 (marzo de 2008).

Los compuestos que se generan cuando los aromas florales se ven alterados químicamente ¿empeoran la contaminación atmosférica? "No hemos pensado aún en esto en [función de] la calidad del aire", dice Fuentes. Agrega que los hallazgos también suscitan inquietudes especiales en relación con el destino de los polinizadores nocturnos, tales como las palomillas, que dependen en gran medida del aroma para encontrar las flores (a diferencia de las abejas, que se valen tanto del color como del aroma durante el día).

Fuentes advierte que no deben aplicarse los nuevos hallazgos al trastorno del colapso de las colonias (CCD, por sus siglas en inglés), un fenómeno inexplicado que ha diezmado a las colonias de abejas en los últimos dos años. Dice que algunas de las mejores evidencias hasta la fecha sugieren que el CCD está más directamente relacionado con los agentes infecciosos y los plaguicidas. Sin embargo, señala, cualquier efecto de la contaminación atmosférica "probablemente constituye un factor adicional de estrés al que las abejas tienen que hacer frente."

Otros comportamientos de insectos que se orientan mediante los aromas químicos, como el apareamiento de los escarabajos, también podrían verse trastornados por la contaminación atmosférica. Por otra parte, algunas plantas pueden beneficiarse del deterioro de las señales transportadas por el aire: "Si los insectos no pueden oler las plantas, no pueden venir a comérselas", dice Jay Evans, un entomólogo investigador del Ministerio de Agricultura de Es- tados Unidos, quien llama al estudio de Fuentes "una hermosa mezcla de buena ecología y química".

Los polinizadores no son la única especie que lidia con los efectos olfativos de la contaminación atmosférica. En el número de Chemical Senses de enero de 2006, Robyn Hudson y sus colegas reportaron que los residentes de la Ciudad de México, una de las más contaminadas del mundo, tenían una capacidad considerablemente menor de detectar y distinguir entre los olores de los alimentos que los residentes de Tlaxcala, un área geográficamente similar con mucha menos contaminación atmosférica. Se observó esta diferencia en múltiples grupos de edades, aun cuando se eliminó del análisis a los fumadores, lo cual es muy sugestivo de un vínculo con los contaminantes que pueden dañar el epitelio olfativo.

Carol Potera

\title{
Señal intensa para los efectos de los teléfonos celulares*
}

$\mathrm{D}$ ado que hay 3 mil millones de usuarios de teléfonos celulares en todo el mundo, y más de $260 \mathrm{mi}-$ llones únicamente en Estados Unidos -entre ellos $46 \%$ de los niños estadounidenses de 8 a 12 años de edad, según las cifras de la empresa Nielsen Mobile publicadas el 10 de septiembre de 2008-, la exposición humana a la radiación de baja energía dentro del rango de 800 a 2000 megahertz está todo el tiempo a su máximo. El intento más reciente de estudiar de manera sistemática la evidencia epi- demiológica del riesgo incrementado de tumores cerebrales relacionado con el uso de teléfonos celulares indica que las repercusiones de este experimento global están saliendo a la luz. En un meta-análisis publicado en el número de mayo de 2008 del International Journal of Oncology, investigadores suecos encontraron asociaciones importantes entre el uso prolongado del teléfono celular y el riesgo de tumores cerebrales.

"Encontramos que el uso de los teléfonos celulares está vinculado a los gliomas [tumores cerebrales malignos] y a los neuromas acústicos [tumores benignos del nervio auditivo del cerebro], y éstos se están manifestando después de tan sólo diez años", dice el autor principal Lennart Hardell, oncólogo y epidemiólogo del cáncer del Hospital de la Universidad de Örebro, Suecia. Específicamente, en los estudios que incluyeron un mínimo de 10 años de exposición, se duplicó el riesgo de gliomas para las exposiciones ipsilaterales (del mismo lado) pero no para las contralaterales

\footnotetext{
* Publicado originalmente en Environmental Health Perspectives, Volumen 116, Número 10, octubre 2008, página A422.
} 
(del lado opuesto) de la cabeza (en relación con cuál mano utilizaba comúnmente el sujeto para sostener su teléfono celular). Se observó un incremento de 2.4 veces en el riesgo de neuromas acústicos debidos a exposición ipsilateral, mientras que no hubo incremento del riesgo de meningiomas (tumores que se presentan en las membranas que cubren el cerebro y la médula espinal).

"Resulta claro que necesitamos más estudios sobre el uso prolongado del teléfono celular para evaluar mejor los riesgos de cáncer", dice el coautor Michael Carlberg. El uso generalizado de los teléfonos celulares data aproximadamente de hace una década, y sin embargo los tumores cerebrales inducidos por las radiaciones normalmente tardan unos 10 a 15 años en desarrollarse, según la Sociedad Americana del Cáncer.

$\mathrm{Al}$ equipo de investigadores de Hardell se deben varios de los estudios incluidos en el meta-análisis. En el número de octubre de 2006 del World Journal of Surgical Oncology, los investigadores reportaron un incremento del 70\% en el riesgo de astrocitomas (tumores cerebrales altamente agresivos) del grado III-IV en los usuarios de teléfonos celulares análogos. Notablemente, no hubo incremento en el riesgo de cáncer testicular, de linfoma de las células $B$ ni de tumores de las glándulas salivales, lo cual sugiere que los hallazgos no se debieron a un sesgo de observación o de memoria, puesto que se habría tenido este sesgo para todos los tipos de tumores.

Con el fin de evitar que los estudios anteriores sesgaran las conclusiones de su meta-análisis de 2008, el equipo omitió del análisis sus propios estudios, y aun así encontró un riesgo significativamente mayor de gliomas y un riesgo incrementado en grado no significativo para los neuromas acústicos (incrementos de $50 \%$ y $210 \%$, respectivamente) para las exposiciones ipsilaterales. "Estamos viendo ahora un patrón consistente

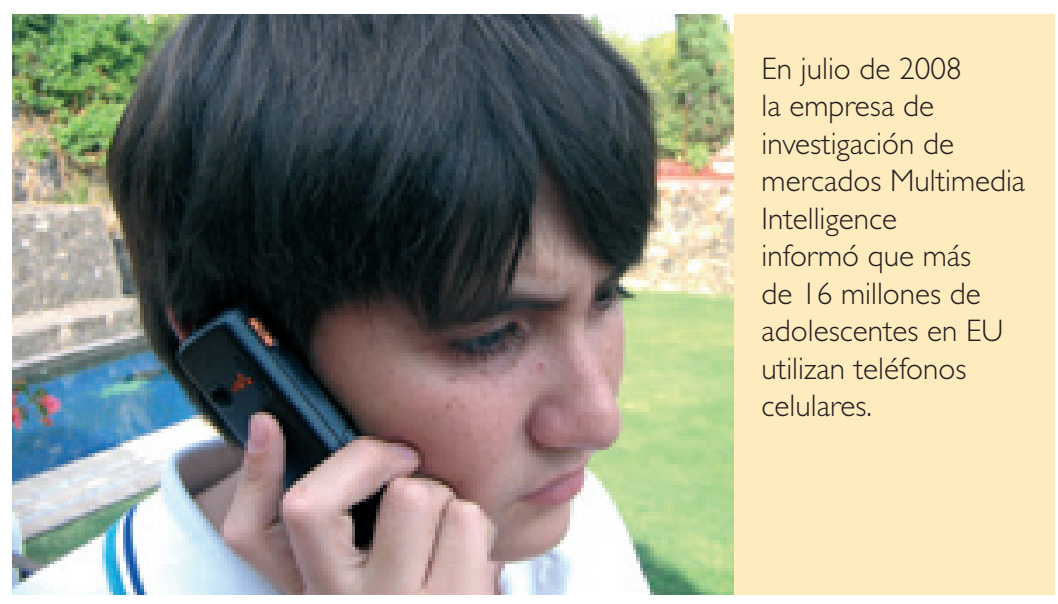

de riesgo incrementado de glioma y neuroma acústico", dice el coautor Kjell Hansson Mild, físico especialista en radiaciones de la Universidad de Umeå, Suecia. "No sólo nuestros propios estudios están demostrando esto, sino también otros estudios que han incluido por lo menos diez años como periodo de latencia."

Está surgiendo evidencia que sugiere que los niños pueden ser más vulnerables a los efectos carcinogénicos potenciales de los teléfonos celulares y de otras tecnologías emisoras de microondas. "Se han suscitado inquietudes sobre la vulnerabilidad potencial de los niños a los campos de radiofrecuencia (RF) debido a que sus sistemas nerviosos en desarrollo son potencialmente más susceptibles", dice Leeka Kheifets, profesora de epidemiología de la Universidad de California en Los Ángeles y anteriormente directora del programa de investigación sobre el Campo Electromagnético del Instituto de Investigación de la Energía Eléctrica. "Además, su tejido cerebral es más conductor, la penetración de la RF es mayor en relación con el tamaño de la cabeza y a lo largo de su vida tendrán una exposición más prolongada [si bien el grado del riesgo de cualquier carcinógeno se determina principalmente con base en el tiempo exacto y la magnitud precisa de la exposición]."

La importancia de tener un cráneo más delgado y de la diferencia en las propiedades dieléctricas se ve confirmada por un estudio publicado en el número de Physics in Medicine and Biology del 7 de junio de 2008, que demuestra que el cerebro de un niño absorbe hasta el doble de RF que un cerebro adulto. Los niños hoy en día experimentan un periodo de exposición más prolongado porque comienzan a utilizar los teléfonos celulares a una edad más temprana, según Hardell; esto podría ser importante, porque la dosis acumulada parece ejercer una fuerte influencia sobre el incremento del riesgo de tumores cerebrales. Sin embargo, Kheifets añade que "faltan datos sobre los efectos de la exposición sobre los tumores cerebrales en niños [y] también es necesario examinar otros efectos sobre la salud."

La industria de las comunicaciones inalámbricas mira con cautela esta investigación. "El peso de la evidencia científica y las conclusiones de un gran número de revisiones científicas de expertos muestran que los teléfonos inalámbricos no plantean ningún riesgo para la salud", dice Joseph Farren, vicepresidente adjunto de asuntos públicos de la Asociación Internacional para las Comunicaciones Inalámbricas (CTIA). "La industria apoya la investigación continua conforme sigue evolucionando la tecnología, pero desea hacer énfasis en el hecho de que existe un consenso entre las 
principales organizaciones de la salud sobre el hecho de que la investigación científica publicada no muestra ningún motivo de preocupación."

Hardell concede que es demasiado pronto para determinar un límite seguro para el uso del teléfono celular. “ ¿Podemos decir que una llamada de diez minutos es equivalente a diez llamadas de un minuto?", pregunta. "Mientras no podamos responder estas preguntas, no podemos establecer un nuevo límite, ni siquiera afirmar qué parámetros o unidades contribuyen a definir ese límite. No obstante, puesto que sí vemos un incremento del riesgo de tumores cerebrales, es necesario aplicar el principio precautorio en esta situación, en especial para las exposiciones prolongadas que tienen probabilidades de afectar a los niños en particular." En la práctica, esto podría implicar limitar el uso de los teléfonos celulares por los niños y emplear teléfonos con altoparlantes para minimizar la exposición directa de la cabeza.

\section{Nathaniel Mead}

\section{¿Una dosis de protección?**}

工os os ácidos grasos poliinsaturados Omega-3 -de los cuales se cree que reducen el riesgo de muchos padecimientos crónicos, incluyendo artritis, cáncer, enfermedades del corazón y pérdida de la memoria-podrían también contribuir a proteger al corazón contra ciertos efectos nocivos de la contaminación atmosférica. En un nuevo estudio realizado por un equipo internacional de investigadores, se asoció la suplementación con Omega-3 a una considerable reducción del estrés cardiaco causado por partículas suspendidas de un diámetro menor de $2.5 \mu \mathrm{m}\left(\mathrm{PS}_{2.5}\right)$ en un grupo de individuos de edad avanzada en la Ciudad de México. ${ }^{1}$ Este estudio es el primero en examinar los efectos de los Omega-3 sobre los biomarcadores de respuesta celular al estrés oxidativo de la contaminación atmosférica.

La exposición a niveles elevados de partículas provenientes del escape de los vehículos y de las emisiones industriales incrementa el riesgo de hipertensión, arritmia cardiaca, infarto y derrame cerebral, a los cuales la población de edad avanzada es más vulnerable. Algunos de los autores habían demostrado ya anteriormente que las $\mathrm{PS}_{2.5}$ promueven las enfermedades del corazón al disminuir la variabilidad en el ritmo cardiaco y que la suplementación con Omega-3 incrementa la variabilidad del ritmo cardiaco. El propósito del estudio en curso era dilucidar de qué manera logran sus efectos los Omega-3.

La población de estudio de 52 residentes de un asilo para ancianos estaba expuesta de manera crónica a niveles elevados de $\mathrm{PS}_{2.5}$; entre los niveles de PS dentro del asilo, donde los residentes pasaban casi todo su tiempo, y el ambiente contaminado del exterior, existía una correlación. Durante cuatro meses a partir del año 2001, la mitad de los participantes en este estudio doble ciego recibieron suplementos de aceite de pescado en dosis típicas para los usuarios de suplementos que se venden sin receta médica; la otra mitad recibió suplementos de aceite de soya.

El equipo de investigación comparó muestras de sangre tomadas de los sujetos antes y durante la suplementación, y encontró que el uso de los Omega-3 estaba asociado a una disminución del daño oxidativo en las células sanguíneas. El efecto antioxidante de los Omega-3 observado fue mucho mayor en aquellos que tomaron el aceite de pescado que en los que tomaron el aceite de soya, diferencia que los investigadores atribuyen a la diversidad de las cantidades y tipos de Omega-3 entre ambas variedades de suplementos (ácido docosahexenoico y ácido eicosapentenoico en el aceite de pescado, y ácido $\alpha$-linoleico en el aceite de soya).

Los autores señalan las limitaciones de su estudio, tales como el hecho de que la muestra es pequeña y que la evaluación de la exposición es limitada. Sin embargo, el hallazgo de que los Omega-3 parecen ser eficaces contra el estrés oxidativo relacionado con la exposición a $\mathrm{PS}_{2.5}$ y de que los suplementos de aceite de pescado ofrecen mayor protección que los de aceite de soya amerita la realización de más estudios sobre una población más amplia.

\section{Cynthia Washam}

\section{Referencias}

I. Romieu I, Garcia-Esteban R, Sunyer J, Rios C, Alcaraz-Zubeldia M, Ruiz Velasco S, Holguin F. The effect of supplementation with Omega-3 polyunsaturated fatty acids on markers of oxidative stress in elderly exposed to $\mathrm{PM}_{2.5}$. Environ Health Perspect I 16:1237-1242.

\footnotetext{
* Publicado originalmente en Environmental Health Perspectives, Volumen 116, Número 9, septiembre 2008, página A394.
} 